\title{
MULTIDIMENSIONAL RESIDUES AND IDEAL MEMBERSHIP
}

\author{
Alessandro Perotti
}

\begin{abstract}
Let $I(f)$ be a zero-dimensional ideal in $\mathbf{C}\left[z_{1}, \ldots, z_{n}\right]$ defined by a mapping $f$. We compute the logarithmic residue of a polynomial $g$ with respect to $f$. We adapt an idea introduced by Aizenberg to reduce the computation to a special case by means of a limiting process.

We then consider the total sum of local residues of $g$ w.r.t. $f$. If the zeroes of $f$ are simple, this sum can be computed from a finite number of logarithmic residues. In the general case, you have to perturb the mapping $f$.

Some applications are given. In particular, the global residue gives, for any polynomial, a canonical representative in the quotient space $\mathbf{C}[z] / I(f)$.
\end{abstract}

\section{Introduction}

We present some algebraic applications of the theory of multidimensional residues in $\mathbf{C}^{n}$. The logarithmic residues and the local (or Grothendieck) residues have been studied by many authors. In particular, we consider some ideas of Aizenberg, Tsikh and Yuzhakov (see [3] or $[\mathbf{6}]$ for a survey).

Let $I(f)$ be a zero-dimensional ideal in $\mathbf{C}\left[z_{1}, \ldots, z_{n}\right]$ defined by a polynomial mapping $f$. In Section 2 we consider the problem of computing the logarithmic residue of a polynomial $g$ with respect to $f$. In the special case when the principal part of every component $f_{i}$ is a power $z_{i}^{k_{i}}$, we give a method in order to simplify the computation. We reduce it to

The author is a member of the G.N.S.A.G.A. of C.N.R.

Keywords. Multidimensional Residues, Local Residues, Integral Representations.

1991 Mathematics subject classifications: Primary: 32A27; Secondary: 32C30, 32A25. 
the application, to only one special polynomial, of a linear functional introduced by Aizenberg $[\mathbf{1}]$ and to the finding of the projection of $g$ onto a finite-dimensional subspace of $\mathbf{C}\left[z_{1}, \ldots, z_{n}\right]$. We also give a description of the radical of $I$.

In the general case, we adapt an idea introduced by Aizenberg to reduce to the special case by means of a limiting process (Proposition 2 and Theorem 1).

In Section 3 we consider the total sum of local residues of a polynomial with respect to the mapping $f$. If all the zeroes of $f$ are simple, we show that this sum can be computed from a finite number of logarithmic residues. In the general case, you have to perturb the mapping $f$ to get a similar result (Theorem 2).

In Section 4 we say something about the applications of these results. In particular, we show (Proposition 3) how the total sum of residues gives, for any polynomial, a canonical representative of its class in the quotient space $\mathbf{C}[z] / I(f)$.

We wish to acknowledge the hospitality of the Mathematics Department of the Trento University.

\section{Logarithmic Residues}

2.1. Let $I=I(f)=\left(f_{1}, \ldots, f_{n}\right)$ be a zero-dimensional polynomial ideal in $\mathbf{C}[z]=\mathbf{C}\left[z_{1}, \ldots, z_{n}\right]$. This means that the zero set $V(f)=$ $V\left(f_{1}, \ldots, f_{n}\right)$ is a discrete algebraic variety in $\mathbf{C}^{n}$, with at most $\operatorname{deg}\left(f_{1}\right) \cdots \operatorname{deg}\left(f_{n}\right)$ points, counted with their multiplicities. Let $z^{(1)}, \ldots, z^{(N)}$ be these (possibly repeated) points. Given a polynomial $g \in \mathbf{C}[z]$, we want to compute the logarithmic residue of $g$ with respect to the mapping $f=\left(f_{1}, \ldots, f_{n}\right)$, that is the sum

$$
\operatorname{LRes}_{f}(g)=\sum_{\nu=1}^{N} g\left(z^{(\nu)}\right) .
$$

2.2. We first consider the special case when $f_{i}=z_{i}^{k_{i}}+P_{i}, i=1, \ldots, n$, where the total degree of $P_{i}$ is less than $k_{i}$. In this situation, the logarithmic residue is given by an explicit formula introduced by Aizenberg (see $[\mathbf{1}],[\mathbf{3}],[\mathbf{4}],[\mathbf{6}]$ ), which can be derived from the application of the Leray-Koppelman integral representation formula for holomorphic functions (see for example [4, Section 3]) on a pseudoball in $\mathbf{C}^{n}$ :

$$
\operatorname{LRes}_{f}(g)=\mathcal{N}\left(g J \frac{z_{1} \cdots z_{n}}{z_{1}^{k_{1}} \cdots z_{n}^{k_{n}}} \sum_{|\alpha|=0}^{\operatorname{deg}(g)}(-1)^{|\alpha|}\left(\frac{P_{1}}{z_{1}^{k_{1}}}\right)^{\alpha_{1}} \cdots\left(\frac{P_{n}}{z_{n}^{k_{n}}}\right)^{\alpha_{n}}\right)
$$


where $J$ is the Jacobian determinant of the mapping $f$ and $\mathcal{N}$ is the linear functional on the polynomials in $z_{1}, \ldots, z_{n}$ and $1 / z_{1}, \ldots, 1 / z_{n}$ that assigns to each polynomial its free term.

We show that the computation of $\operatorname{LRes}_{f}(g)$ can be simplified by exploiting the decomposition $\mathbf{C}[z]=\mathbf{C}_{k-1}[z] \oplus I$, where $\mathbf{C}_{k-1}[z]$ is the $N$-dimensional space of the polynomials in $\mathbf{C}[z]$ with degree less than $k_{i}$ with respect to $z_{i}$ for every $i=1, \ldots, n$. This follows from the particular form of the polynomials $f_{i}$. In fact, it can be easily seen that $f_{1}, \ldots, f_{n}$ is a Gröbner basis (not necessarily reduced) of the ideal $I$ with respect to any degree ordering.

Let $z^{\alpha}$ denote the monomial $z_{1}^{\alpha_{1}} \cdots z_{n}^{\alpha_{n}}$. Let $K_{0}(z, \zeta) \in \mathbf{C}[z, \zeta]$ be a polynomial which belongs to $\mathbf{C}_{k-1}[z]$ for any fixed $\zeta$ and to $\mathbf{C}_{k-1}[\zeta]$ for any fixed $z$ and has the following property:

(*) The set $\left\{K_{\alpha}(\zeta)\right\}$ defined by the decomposition $K_{0}(z, \zeta)=$ $\sum_{\alpha} K_{\alpha}(\zeta) z^{\alpha}$ is a basis of $\mathbf{C}_{k-1}[\zeta]$.

Let $K(z)=\operatorname{LRes}_{f}\left(K_{0}\right)=\sum_{\alpha} \operatorname{LRes}_{f}\left(K_{\alpha}\right) z^{\alpha}$. Consider the non-degenerate bilinear form on $\mathbf{C}[z]$ defined for any $p=\sum_{\alpha} a_{\alpha} z^{\alpha}, q=\sum_{\alpha} b_{\alpha} z^{\alpha}$ by

$$
\langle p, q\rangle_{K}=\sum_{\alpha, \beta} m_{\alpha, \beta} a_{\alpha} b_{\beta}
$$

where $M=\left(m_{\alpha, \beta}\right)$ is the transition matrix from the basis $\left\{K_{\alpha}\right\}$ to the basis $\left\{z^{\beta}\right\}_{0 \leq \beta_{i}<k_{i}}$.

Then we get the following result.

Proposition 1. The logarithmic residue of $g \in \mathbf{C}[z]$ with respect to $f$ is given by the linear functional $\langle\cdot, K\rangle_{K}$ evaluated on the (unique) projection $g_{0}$ of $g$ in $\mathbf{C}_{k-1}[z]$.

Proof: If $g=g_{0}+g_{1} \in \mathbf{C}_{k-1}[z] \oplus I$ and $g_{0}=\sum_{\alpha} a_{\alpha} z^{\alpha}=\sum_{\alpha, \beta} m_{\alpha, \beta} a_{\alpha} K_{\beta}$, then $\operatorname{LRes}_{f}(g)=\operatorname{LRes}_{f}\left(g_{0}\right)=\sum_{\alpha, \beta} m_{\alpha, \beta} a_{\alpha} \operatorname{LRes}_{f}\left(K_{\beta}\right)=\left\langle g_{0}, K\right\rangle_{K}$.

Two possible choices for the kernel $K_{0}(z, \zeta)$ are the following:

(i) $K_{0}(z, \zeta)=\sum_{0<\alpha_{i}<k_{i}} \prod_{i}\left(z_{i} \zeta_{i}\right)^{\alpha_{i}}$, with associated form $\langle p, q\rangle=$ $\sum_{\alpha} a_{\alpha} b_{\alpha}$

(ii) $K_{0}(z, \zeta)=\prod_{i} \frac{\left(\zeta_{i}^{k_{i}}-z_{i}^{k_{i}}\right)}{\left(\zeta_{i}-z_{i}\right)}$, with associated form $\langle p, q\rangle=\sum_{\alpha} a_{\alpha} b_{k-\alpha-1}$, where $k-\alpha-1$ is the multiindex $\left(k_{1}-\alpha_{1}-1, \ldots, k_{n}-\alpha_{n}-1\right)$.

Remark. The second kernel is a Hefer determinant of the mapping $Q=f-P=\left(z_{1}^{k_{1}}, \ldots, z_{n}^{k_{n}}\right)$. It is the determinant of the polynomial 
matrix $\left(P_{i j}(z, \zeta)\right)$ defined by the Hefer expansions

$$
Q_{i}(\zeta)-Q_{i}(z)=\sum_{j} P_{i j}(z, \zeta)\left(\zeta_{j}-z_{j}\right)
$$

Remark. If $K_{0}$ have integer coefficients, then the coefficients of $K(z)$ are integer polynomial expressions in the coefficients of the $f_{i}$. If the $f_{i}$ have integer, rational or real coefficients respectively, the same holds for $K(z)$.

2.3. Let $K_{0}(z, \zeta)$ be the kernel given in (i). If the polynomials $f_{i}$ have real coefficients, then $\langle K, K\rangle_{K}$ is a real number greater than $N^{2}$, since $K(0)=N$. It follows the decomposition $\mathbf{C}[z]=\langle K\rangle \oplus$ $\left(\mathbf{C}_{k-1}[z] \cap\langle K\rangle^{\perp}\right) \oplus I$, where the second subspace is formed by the polynomials $g \in \mathbf{C}_{k-1}[z]$ such that $\operatorname{LRes}_{f}(g)=0$. Then the set of polynomials vanishing on $V(f)$, that is the radical ideal $\operatorname{Rad} I$, decomposes as

$$
\operatorname{Rad} I=\left(\operatorname{Rad} I \cap \mathbf{C}_{k-1}[z]\right) \oplus I
$$

with

$$
\begin{aligned}
\operatorname{Rad} I \cap \mathbf{C}_{k-1}[z]=\left\{g \in\langle K\rangle^{\perp} \cap \mathbf{C}_{k-1}[z]:\right. & \left(g^{l}\right)_{0} \in\langle K\rangle^{\perp} \\
& \text { for every } l=2, \ldots, N\} .
\end{aligned}
$$

Here $\left(g^{l}\right)_{0}$ denotes the component of $g^{l}$ in $\mathbf{C}_{k-1}[z]$.

Remark. Since $\left\langle K_{0}(a, \zeta), K(\zeta)\right\rangle_{K}=K(a)$, if $K$ is not the constant $N$ we get that $K_{0}(a, \zeta) \in\langle K\rangle^{\perp} \cap \mathbf{C}_{k-1}[\zeta]$ if and only if $K(a)=0$.

2.4. Now we return to the general case. Let $f=\left(f_{1}, \ldots, f_{n}\right)$ be a polynomial mapping with a discrete zero set $V(f)=\left\{z^{(1)}, \ldots, z^{(N)}\right\}$. Let $k_{i}=\operatorname{deg}\left(f_{i}\right)$ for $i=1, \ldots, n$. Then $N \leq k_{1} \cdots k_{n}$.

We use an idea introduced by Aizenberg to reduce the general case to the previous case.

If, for some $i$, the polynomial $f_{i}$ has the special form considered in Section 2.1, with principal part $z_{j}^{k_{i}}$, we set $f_{j}^{\prime}=f_{i}$. For the remaining indices, we set $f_{i}^{\prime}=z_{i}^{k_{i}+1}+\mu f_{i}, \mu \in \mathbf{C}$. Let $I_{\mu}^{\prime}$ be the ideal generated by $f_{1}^{\prime}, \ldots, f_{n}^{\prime}$. It has zero set $V\left(f^{\prime}\right)$ containing $M=\operatorname{deg}\left(f_{1}^{\prime}\right) \cdots \operatorname{deg}\left(f_{n}^{\prime}\right)$ points (with multiplicities), which we shall denote by $z_{\mu}^{(1)}, \ldots, z_{\mu}^{(M)}$. If $f$ is not in the special form, than $M>N$. 
Let $g \in \mathbf{C}[z]$. Let $a=\left(a_{1}, \ldots, a_{n}\right)$ be a vector of complex parameters and $g^{\prime}=g+\sum_{i} a_{i} z_{i}$. For any fixed value of $\mu, f^{\prime}$ has the special form considered in 2.1. Then we can compute the logarithmic residues $\operatorname{LRes}_{f^{\prime}}\left(\left(g^{\prime}\right)^{l}\right), l=1 \ldots, M$. These are polynomial expressions in $\mu, a_{1}, \ldots, a_{n}$. From Newton's formula, we can find the elementary symmetric functions $\sigma_{g^{\prime}}^{l}(\mu)$ in the quantities $g^{\prime}\left(z_{\mu}^{(1)}\right), \ldots, g^{\prime}\left(z_{\mu}^{(M)}\right)$.

It follows from Rouché's principle (see [4, Section 2]) that $N$ elements of $V\left(f^{\prime}\right)$ tend to the points in $V(f)$ as $\mu \rightarrow \infty$, while the other $M-N$ points tend to $\infty$. After reordering, we can assume that $z_{\mu}^{(1)}, \ldots, z_{\mu}^{(N)}$ have limits $z^{(1)}, \ldots, z^{(N)}$ respectively.

Let us denote by $\sigma_{g^{\prime}}^{l}, l=1, \ldots, N$, the elementary symmetric functions in $g^{\prime}\left(z^{(1)}\right), \ldots, g^{\prime}\left(z^{(N)}\right)$. The polynomial $g^{\prime}$ can vanish identically (with respect to $a$ ) only in the point 0 and in this case $g(0)=0$. If $0 \in V(f)$, then $0 \in V\left(f^{\prime}\right)$ with the same multiplicity $h$. Assume that $z_{\mu}^{(1)}=0, \ldots, z_{\mu}^{(h)}=0$. Let us denote by $\sigma_{g^{\prime}}^{-l}, l=1, \ldots, N-h$, the elementary symmetric functions in $g^{\prime}\left(z^{(h+1)}\right)^{-1}, \ldots, g^{\prime}\left(z^{(N)}\right)^{-1}$.

Proposition 2. (i) $\sigma_{g}^{l}=\lim _{a \rightarrow 0} \sigma_{g^{\prime}}^{l}$ for every $l=1, \ldots, N$;

(ii) $\sigma_{g^{\prime}}^{l}=\lim _{\mu \rightarrow \infty} \frac{\sigma_{g^{\prime}}^{M-N+l}(\mu)}{\sigma_{g^{\prime}}^{M-N}(\mu)}$ for every $l=1, \ldots, N$.

Proof: (i) is immediate, since $\sigma_{g^{\prime}}^{l}$ depends polynomially from $a$; for (ii), we adapt the arguments given in [4, Section 21.3]. If $0 \notin V(f)$ then $\sigma_{g^{\prime}}^{M}(\mu) \not \equiv 0$. For all $a$ with the exception of a set of complex dimension $n-1$, the ratios $\sigma_{g^{\prime}}^{M-l}(\mu)\left(\sigma_{g^{\prime}}^{M}(\mu)\right)^{-1}$ tend to 0 for $l=N+1, \ldots, M$, and to $\sigma_{g^{\prime}}^{-l}$ for $l=1, \ldots, N$. But the functions $\sigma_{g^{\prime}}^{l}(\mu)$ are polynomials in $\mathbf{C}(a)[\mu]$ and therefore the ratios $\sigma_{g^{\prime}}^{M-l}(\mu)\left(\sigma_{g^{\prime}}^{M}(\mu)\right)^{-1}$ have limit in $\mathbf{C}(a)$, as $\mu \rightarrow \infty$, equal to 0 for $l=N+1, \ldots, M$, and equal to $\sigma_{g^{\prime}}^{-l}$ for $l=1, \ldots, N$.

Then $\sigma_{g^{\prime}}^{M-N+l}(\mu)\left(\sigma_{g^{\prime}}^{M-N}(\mu)\right)^{-1}$ tends to $\sigma_{g^{\prime}}^{-N+l}\left(\sigma_{g^{\prime}}^{-N}\right)^{-1}=\sigma_{g^{\prime}}^{l}$ for every $l=1, \ldots, N$.

If $0 \in V(f)$ with multiplicity $h$, then $\sigma_{g^{\prime}}^{l}(\mu) \equiv 0$ for $l=M-h+$ $1, \ldots, M$, while $\sigma_{g^{\prime}}^{M-h}(\mu) \not \equiv 0$. The ratios $\sigma_{g^{\prime}}^{M-h-l}(\mu)\left(\sigma_{g^{\prime}}^{M-h}(\mu)\right)^{-1}$ tend to 0 for $l=N-h+1, \ldots, M-h$, and to $\sigma_{g^{\prime}}^{-l}$ for $l=1, \ldots, N-h$. In particular, $\sigma_{g^{\prime}}^{M-N}(\mu)\left(\sigma_{g^{\prime}}^{M-h}(\mu)\right)^{-1}$ has limit $\sigma_{g^{\prime}}^{-N+h} \not \equiv 0$, hence $\sigma_{g^{\prime}}^{M-N}(\mu) \not \equiv 0$. 
It remains to note that $\sigma_{g^{\prime}}^{l}=\sigma_{g^{\prime}}^{-N+h+l}\left(\sigma_{g^{\prime}}^{-N+h}\right)^{-1}$ for every $l=1, \ldots, N-h$.

Remark. In general, the number $N$ is not known in advance. It can be determined from the previous limiting processes, by counting how many ratios $\sigma_{g^{\prime}}^{M-h-l}(\mu)\left(\sigma_{g^{\prime}}^{M-h}(\mu)\right)^{-1}$ tend to 0 . Equivalently, it is the number of functions $\sigma_{g^{\prime}}^{M-h-l}(\mu)$ which have the same $\mu$-degree as $\sigma_{g^{\prime}}^{M-h}(\mu)$.

In particular, $\sigma_{g}^{1}=\operatorname{LRes}_{f}(g)$. We have proved the following result.

Theorem 1. The logarithmic residue of any $g \in \mathbf{C}[z]$ with respect to $f$ can be computed from

$$
\operatorname{LRes}_{f}(g)=\lim _{a \rightarrow 0} \lim _{\mu \rightarrow \infty} \frac{\sigma_{g^{\prime}}^{M-N+1}(\mu)}{\sigma_{g^{\prime}}^{M-N}(\mu)} .
$$

\section{Local Residues}

Now we consider the total sum of local residues of a polynomial $g \in \mathbf{C}[z]$ with respect to the polynomial mapping $f=\left(f_{1}, \ldots, f_{n}\right)$. In general, if $f=\left(f_{1}, \ldots, f_{n}\right)$ is a holomorphic mapping with an isolated zero $a$ in a closed neighbourhood $U_{a}$ of $a$, the local (or Grothendieck) residue at $a$ of a holomorphic function $g$ on $U_{a}$ with respect to $f$ is the integral

$$
\operatorname{res}_{a, f}(g)=\frac{1}{(2 \pi i)^{n}} \int_{\Gamma_{a}(f)} \frac{g d z_{1} \wedge \cdots \wedge d z_{n}}{f_{1} \cdots f_{n}}
$$

where $\Gamma_{a}(f)$ is the $n$-chain $=\left\{z \in U_{a}:\left|f_{i}(z)\right|=\epsilon_{i}, i=1, \ldots, n\right\}$, with $\epsilon_{i}>0$ such that $\Gamma_{a}(f)$ is relatively compact in $U_{a}$ (see for example [5]).

3.1. Let $I=\left(f_{1}, \ldots, f_{n}\right)$ be a zero-dimensional polynomial ideal in $\mathbf{C}[z]$. Since $f$ has a finite number of isolated zeroes, we can consider the global residue $\operatorname{Res}_{f}(g)=\sum_{a \in V(f)} \operatorname{res}_{a, f}(g)$ of the local residues of $g \in \mathbf{C}[z]$ with respect to $f$.

Remark. If $g=h \cdot J$, where $J$ is the Jacobian determinant of the mapping $f$, the local residue coincides with the logarithmic residue of $h$ at $a$. Then $\operatorname{LRes}_{f}(h)=\operatorname{Res}_{f}(h \cdot J)$.

If $f$ has the special form $f_{i}=z_{i}^{k_{i}}+P_{i}$, with $\operatorname{deg}\left(P_{i}\right)<k_{i}$, the global residue $\operatorname{Res}_{f}(g)$ can be computed from the explicit formula of Aizenberg $[\mathbf{1}]$. 
In the general case, Yuzhakov introduced in $[\mathbf{9}]$ an algorithm to reduce the problem to the special case, by applying the transformation formula for the local residue and the generalized resultants. We proceed in a different way. We obtain $\operatorname{Res}_{f}(g)$ from the computation of a finite number of (global) logarithmic residues, which can be found with the method of Section 2 .

3.2. In the case that the zeroes $z^{(1)}, \ldots, z^{(N)}$ of $f$ are all simple, then $\operatorname{Res}_{f}(g)=\sum_{\nu=1}^{N} \frac{g\left(z^{(\nu)}\right)}{J\left(z^{(\nu)}\right)}$. We can now apply the following lemma, which generalizes Newton's formulas (for a proof, see for example [7]).

Lemma 1. Let $\sigma^{l}(a)$ denote the $l$-th elementary symmetric function of $m$ scalars $a_{1}, \ldots, a_{m}$. If $b_{1}, \ldots, b_{m}$ are scalars different from zero, the sum $\sigma^{1}\left(\frac{a}{b}\right)=\frac{a_{1}}{b_{1}}+\cdots+\frac{a_{m}}{b_{m}}$ is given by

$$
\sigma^{1}\left(\frac{a}{b}\right)=\sum_{k=0}^{m-1}(-1)^{k} \frac{\sigma^{1}\left(a b^{k}\right) \cdot \sigma^{m-k-1}(b)}{\sigma^{m}(b)} .
$$

Then we obtain the following formula:

$$
\operatorname{Res}_{f}(g)=\sum_{k=0}^{N-1}(-1)^{k} \frac{\sigma_{g \cdot J^{k}}^{1} \cdot \sigma_{J}^{N-k-1}}{\sigma_{J}^{N}}
$$

where $\sigma_{g \cdot J^{k}}^{1}$ and $\sigma_{J}^{l}$ can be found from Proposition 2 .

3.3. If not all the zeroes of $f$ are simple, $f$ can be perturbed. We consider $f-w$, where $w$ is a small complex $n$-tuple. For generic values of $w$, the Jacobian $J$ does not vanish at the zeroes of $f-w$. Let $z^{(1)}(w), \ldots, z^{(N)}(w)$ be the elements of $V(f-w)$. In [6, Section 6.2], Tsikh showed that the sum

$$
\phi(w)=\sum_{\nu=1}^{N} \frac{g\left(z^{(\nu)}(w)\right)}{J\left(z^{(\nu)}(w)\right)}
$$

is a holomorphic function in $w$ on a small neighbourhood of 0 . Then $\phi(0)$ is the sum of the local residues of $g$ at the zeroes of $f$. As a result, we obtain the following theorem.

Theorem 2. The global residue $\operatorname{Res}_{f}(g)$ of any $g \in \mathbf{C}[z]$ with respect to $f$ is equal to $\psi(0)$, where $\psi(w)$ is the holomorphic function given by

$$
\psi(w)=\sum_{k=0}^{N-1}(-1)^{k} \frac{\operatorname{LRes}_{f-w}\left(g \cdot J^{k}\right) \cdot \sigma_{J}^{N-k-1}(w)}{\sigma_{J}^{N}(w)} .
$$


Here $\sigma_{J}^{l}(w)$ are the elementary symmetric functions in

$$
J\left(z^{(1)}(w)\right), \ldots, J\left(z^{(N)}(w)\right),
$$

which can be found from the logarithmic residues $\operatorname{LRes}_{f-w}\left(J^{l}\right)$, $l=1, \ldots, N$.

\section{Applications}

4.1. The global residues and the total logarithmic residues have well known applications. They give a method for eliminating variables which does not use resultants. For any $i=1, \ldots, n$, from $\operatorname{LRes}_{f}$ a univariate polynomial in $I(f) \cap \mathbf{C}\left[z_{i}\right]$ of degree $N$ can be computed. It preserves multiplicities of the zeroes of $f$ (for this method, see [4, Section 21]).

From $\operatorname{Res}_{f}$ a membership criterion for the ideal $I(f)$ can be deduced. In [8], Tsikh applied Lasker-Noether Theorem and got the following:

$$
\begin{array}{r}
g \in I(f) \Leftrightarrow \operatorname{Res}_{f}(g(\zeta) H(z, \zeta))=0, \\
\text { where } H(z, \zeta) \text { is a Hefer determinant of } f .
\end{array}
$$

Remark. A polynomial Hefer determinant of $f$ can be computed from the Hefer expansions

$$
f_{i}(\zeta)-f_{i}(z)=\sum_{j} P_{i j}(z, \zeta)\left(\zeta_{j}-z_{j}\right)
$$

where $P_{i j}(z, \zeta)=\frac{f_{i}\left(\zeta_{1}, \ldots, \zeta_{j}, z_{j+1}, \ldots, z_{n}\right)-f_{i}\left(\zeta_{1}, \ldots, \zeta_{j-1}, z_{j}, \ldots, z_{n}\right)}{\zeta_{j}-z_{j}}$.

Note that from $P_{i j}(z, \zeta)$ and $P_{i j}(\zeta, z)$ we can get a Hefer determinant which is symmetric in $z$ and $\zeta$.

4.2. Let $g, h \in \mathbf{C}[z]$ and

$$
\begin{aligned}
g_{0}(\zeta) & =\operatorname{Res}_{f}(g(z) H(z, \zeta)), \\
h_{0}(\zeta) & =\operatorname{Res}_{f}(h(z) H(z, \zeta)) .
\end{aligned}
$$

From the membership criterion above we get that $g_{0}=h_{0}$ if and only if the difference $g-h \in I(f)$, that is $g$ and $h$ define the same class in the $N$-dimensional quotient space $\mathbf{C}[z] / I(f)$.

If we apply the transformation formula for the global residue (see $[\mathbf{8}]$ ) to the Hefer expansion of $f$, we get, for any polynomial $p$, 
$\operatorname{Res}_{z-\zeta} p(z)=\operatorname{Res}_{f-f(\zeta)}(p(z) H(z, \zeta))$. It follows that for any $a \in V(f)$, $\operatorname{Res}_{f}(p(z) H(z, a))=p(a)$. In particular, we get $\operatorname{Res}_{f} H(z, a)=1$.

From this we can deduce that

$$
\operatorname{Res}_{f}\left(g_{0}(z) H(z, \zeta)\right)=\operatorname{Res}_{f}(g(z) H(z, \zeta))=g_{0}(\zeta)
$$

For simplicity, assume that the zeroes of $f$ are simple. Then

$$
\begin{aligned}
\operatorname{Res}_{f}\left(g_{0}(z) H(z, \zeta)\right) & =\sum_{\nu} \frac{g_{0}\left(z^{\nu}\right) H\left(z^{\nu}, \zeta\right)}{J\left(z^{\nu}\right)} \\
& =\sum_{\nu, \mu} \frac{g\left(z^{\mu}\right) H\left(z^{\nu}, z^{\mu}\right) H\left(z^{\nu}, \zeta\right)}{J\left(z^{\nu}\right) J\left(z^{\mu}\right)} \\
& =\sum_{\mu} \frac{g\left(z^{\mu}\right)}{J\left(z^{\mu}\right)} \operatorname{Res}_{f}\left(H\left(z, z^{\mu}\right) H(z, \zeta)\right) \\
& =\operatorname{Res}_{f}(g(z) H(z, \zeta))=g_{0}(\zeta) .
\end{aligned}
$$

As a result, we get the following proposition.

Proposition 3. Let $g \in \mathbf{C}[z], g_{0}(\zeta)=\operatorname{Res}_{f}(g(z) H(z, \zeta))$. Then $g-g_{0} \in I(f)$, that is $g_{0}$ represents $g$ in the quotient space $\mathbf{C}[z] / I(f)$. In particular, $\operatorname{Res}_{f} g=\operatorname{Res}_{f} g_{0}$.

Note added in proof. The paper by E. Cattani, A. Dickenstein, B. Sturmfels, Computing multidimensional residues, Algorithms in Algebraic Geometry and Applications (L. Gonzales-Vega and T. Recio, eds.), Progress in Mathematics, Vol. 143, Birkhäuser Verlag, Basel, 1996, pp. 135-164, contains interesting relations between global residues and Gröbner bases and other references about these problems.

\section{References}

1. L. A. Aizenberg, On a formula for the generalized multidimensional logarithmic residue and solutions of systems of nonlinear equations, Dokl. Akad. Nauk SSSR 234 (1977), 505-508; English transl., Soviet Math. Dokl. 18 (1977), 691-695.

2. L. A. Aizenberg And A. K. Tsikh, Application of the multidimensional logarithmic residue to systems of nonlinear algebraic equations, Sibirsk. Mat. Zh. 20 (1979), 699-707; English transl., Siberian Math. J. 20 (1979), 485-491. 
3. L. A. Aizenberg, A. K. Tsikh and A. P. Yuzhakov, Multidimensional Residues and Applications, in "Several Complex Variables II," (G. M. Khenkin and A. G. Vitushkin, eds.), Encyclopaedia of Math. Sc. 8, Springer-Verlag, Berlin, Heidelberg, New-York, 1990, pp. $1-58$.

4. L. A. Aizenberg And A. P. Yuzhakov, "Integral Representations and Residues in Multidimensional Complex Analysis," Nauka, Novosibirsk, 1979; English transl., Translations of Mathematical Monographs 58, Amer. Math. Soc., Providence, 1983.

5. P. Griffiths and J. Harris, "Principles of Algebraic Geometry," Wiley, New York, Chichester, 1978.

6. A. K. Tsikn, "Multidimensional Residues and Their Applications," Nauka, Novosibirsk, 1988; English transl., Translations of Mathematical Monographs 103, Amer. Math. Soc., Providence, 1992.

7. A. K. TsikH, "Residues with respect to holomorphic mappings and their applications," Krasnoyarsk Univ. Press, Krasnoyarsk, 1986, (Russian).

8. A. K. Tsikh and A. P. Yuzhakov, Properties of the total sum of residues with respect to a polynomial map and applications of them, Sibirsk. Mat. Zh. 25 (1984), 207-213; English transl., Siberian Math. J. 25 (1984), 677-682.

9. A. P. Yuzhakov, On the computation of the total sum of residues relative to a polynomial mapping in $\mathbf{C}^{n}$, Dokl. Akad. Nauk SSSR 275 (1984), 817-820; English transl., Soviet Math. Dokl. 29 (1984), $321-324$.

\author{
Dipartimento di Matematica \\ Università di Milano \\ Via Saldini 50 \\ I-20133 Milano \\ ITALY \\ e-mail: perotti@vmimat.mat.unimi.it
}

Primera versió rebuda el 30 de gener de 1997, darrera versió rebuda el 4 de novembre de 1997 